\title{
Recurrent 12q13-15 chromosomal aberrations, high frequency of isocitrate dehydrogenase 1 mutations, and absence of high mobility group AT-hook 2 expression in periosteal chondromas
}

\author{
IOANNIS PANAGOPOULOS ${ }^{1,2}$, LUDMILA GORUNOVA ${ }^{1,2}$, INGEBORG TAKSDAL $^{3}$, \\ BODIL BJERKEHAGEN ${ }^{4}$ and SVERRE HEIM ${ }^{1,2,5}$
}

\begin{abstract}
${ }^{1}$ Section for Cancer Cytogenetics, Institute for Cancer Genetics and Informatics, The Norwegian Radium Hospital, Oslo University Hospital, Oslo 0424; ${ }^{2}$ Centre for Cancer Biomedicine, Faculty of Medicine, University of Oslo, Oslo 0316; Departments of ${ }^{3}$ Radiology and Nuclear Medicine, and ${ }^{4}$ Pathology, The Norwegian Radium Hospital, Oslo University Hospital, Oslo 0424; ${ }^{5}$ Faculty of Medicine, University of Oslo, Oslo 0316, Norway
\end{abstract}

Received July 6, 2014; Accepted February 13, 2015

DOI: $10.3892 / 01.2015 .3197$

\begin{abstract}
Periosteal chondroma is a benign cartilage tumor that accounts for $<2 \%$ of chondromas. In the present study, four cases of periosteal chondromas were cytogenetically investigated and studied for the expression of high-mobility group AT-hook 2 (HMGA2), mutations in codons 132 of isocitrate dehydrogenase (IDH)1 and 172 of $I D H 2$; mutations $-\mathrm{C} 228 \mathrm{~T}$ and $-\mathrm{C} 250 \mathrm{~T}$ in the promoter region of telomerase reverse transcriptase (TERT); and for methylation in the promoter regions of $O$-6-methylguanine-DNA methyltransferase $(M G M T)$ and cellular retinol binding protein 1 (CRBPI). Chromosome aberrations of 12q13-15 were found in two out of the four tumors, while two had a normal karyotype. Two periosteal chondromas carried the mutation IDH1R132C (CGT>TGT), and two carried the mutation IDH1R132L (CGT>CTT). However, none of the four tumors had methylated $M G M T$ and $C R B P 1$ promoters or mutations at codon 172 of IDH2. In addition, -C228T and $-\mathrm{C} 250 \mathrm{~T}$ mutations were not present in the promoter region of TERT, nor was HMGA2 demonstrated to be expressed. The present study indicated that in periosteal chondromas, the involvement of 12q13-15 in structural rearrangements may be recurrent but that $H M G A 2$ is not expressed. Additionally, the periosteal chondromas investigated in the study carried
\end{abstract}

Correspondence to: Dr Ioannis Panagopoulos, Section for Cancer Cytogenetics, Institute for Cancer Genetics and Informatics, The Norwegian Radium Hospital, Oslo University Hospital, Ullernchausséen 70, P.O. Box 4953, Oslo 0424, Norway

E-mail: ioannis.panagopoulos@rr-research.no

Key words: cytogenetics, 12q13-15 chromosomal aberrations, isocitrate dehydrogenase 1 mutations, high-mobility group AT-hook 2, periosteal chondromas a heterozygous IDH1R132 mutation, the $M G M T$ and $C R B P I$ promoters were not methylated, and -C228T and -C250T mutations in the promoter region of TERT were absent.

\section{Introduction}

Periosteal chondroma is a benign cartilage tumor that accounts for $<2 \%$ of chondromas. This type of tumor grows in the periosteal region and frequently erodes the underlying bone cortex. Periosteal chondroma can lead to sclerosis of underlying bone, and the formation of a 'dished' area under the tumor with a 'buttress' or peripheral wall of reactive bone at its edge. The bone cortex may be abnormal or thinned adjacent to the tumor. Periosteal chondromas typically present in the second and third decades of life with a male predilection (2:1), are usually asymptomatic, and are commonly identified incidentally on radiographs obtained for other reasons (1). Common sites of occurrence include the proximal humerus, proximal and distal femur and the phalanges of the hands and feet (1). A tender swelling or the feeling of a mass may bring the lesion to clinical attention. Periosteal chondroma may be confused with chondrosarcoma or periosteal and parosteal osteosarcoma. Therefore, diagnosis requires a thorough investigation that includes radiological examination and biopsy. Simple excision of the tumor is often curative.

Previous studies have described clonal cytogenetic abnormalities in 7 periosteal chondromas, but no specific aberration pattern has been detected (2-4). In other studies, IDH1 mutations were observed in 6 out of the 8 periosteal chondromas examined $(5,6)$. The present study investigated cytogenetic and molecular genetic data in 4 cases of periosteal chondroma.

\section{Materials and methods}

Ethics statement. The study was approved by the regional ethics committee (Regional komité for medisinsk forskningsetikk Sør-Øst, Norge, http://helseforskning.etikkom.no). 
Patients. A total of 3 females and 1 male, between 8 and 47 years of age, were included in the study. Clinical data are presented in Table I.

Chromosome banding analysis. Samples from the surgically resected tumors were mechanically and enzymatically disaggregated and short-term cultured as described previously (6). The cultures were harvested and the chromosomes were G-banded using Wright's stain (Sigma-Aldrich; St Louis, MO, USA) (7). The subsequent cytogenetic analysis and karyotype description followed the recommendations of the International System for Human Cytogenetic Nomenclature (8).

Polymerase chain reaction (PCR) for IDH1 and IDH2 mutations. Genomic DNA was extracted using a Maxwell 16 Research Instrument system and a Maxwell 16 Tissue DNA Purification Kit (Promega Corporation, Madison, WI, USA). For the detection of possible IDHI and IDH2 mutations, quantitative (q)PCR with high resolution melt curve analysis (HRM) was performed followed by Sanger sequencing to confirm positive HRM screens (9). As positive and negative controls, plasmids containing the wild-type IDH1R132 and IDH2R172 and the mutated IDH1R132H and IDH2R172M were used. The 20- $\mu$ l PCR mixture contained $10 \mu \mathrm{l}$ Precision Melt Supermix (Bio-Rad Laboratories AB, Oslo, Norway), $0.2 \mu \mathrm{M}$ of each of the forward and reverse primers (custom-made; Invitrogen Life Technologies, Carlsbad, CA, USA), and $20 \mathrm{ng}$ of genomic DNA. To identify possible IDH1R132 mutations, the following primers were used: The forward primer was IDH1-F1, 5'-TCA GAG AAG CCA TTA TCT GCA-3' and the reverse was IDH1-R1, 5'-AAT CAC ATT ATT GCC AAC ATG A-3'. To identify possible IDH1R172 mutations the following primers were used: The forward was IDH2-F1New, 5'-TAG TCC CTG GCT GGA CCA-3' and the reverse was IDH2-R1New, 5'-TGC CCA GGT CAG TGG ATC-3'. The PCRs were conducted on a CFX96 Touch Real-Time PCR Detection System using the Bio-Rad CFX Manager 2.1 software (Bio-Rad Laboratories AB). The PCR cycling and melt curve conditions were as follows: An initial denaturation at $95^{\circ} \mathrm{C}$ for $2 \mathrm{~min}$ followed by 40 cycles of $10 \mathrm{sec}$ at $95^{\circ} \mathrm{C}, 30 \mathrm{sec}$ at $55^{\circ} \mathrm{C}$ (plus plate read). Next, the melt curve started with denaturation at $95^{\circ} \mathrm{C}$ for $30 \mathrm{sec}$ and annealing at $60^{\circ} \mathrm{C}$ for $1 \mathrm{~min}$. The melt curve program then continued from $65^{\circ} \mathrm{C}$ to $95^{\circ} \mathrm{C}$ with increments of $0.2^{\circ} \mathrm{C}$ for $10 \mathrm{sec}$, plus a final plate read. The HRM of the data was made using the Precision Melt Analysis software, version 1.2 (Bio-Rad Laboratories AB). To confirm positive HRM screens, Sanger sequencing was performed as follows: The PCR products were purified using the NucleoSpin Gel and PCR Clean-up kit (Machery-Nagel $\mathrm{GmbH}$, Düren, Germany) and direct Sanger sequencing was performed using the LIGHTrun Sequencing service (GATC Biotech AG, Konstanz, Germany; www.gatc-biotech. com/en/products/sanger-services/lightrun-sequencing.html). BLAST software (blast.ncbi.nlm.nih.gov/Blast.cgi) was used for computer analysis of the sequence data.

PCR for-C228T and-C250T mutations. In order to detect the possible-C228T and -C250T mutations in the promoter region of telomerase reverse transcriptase (TERT), which correspond to positions 124 and $146 \mathrm{bp}$, respectively, upstream of the TERT
ATG start site (10), PCR was conducted as follows: The $25 \mu \mathrm{l}$ PCR volume contained 1X PrimeSTAR GXL Buffer (Takara Bio Europe SAS, Saint-Germain-en-Laye, France), $200 \mu \mathrm{M}$ of each dNTP, $0.4 \mu \mathrm{M}$ of each of the primers (custom-made; Invitrogen Life Technologies), 1.25 units of PrimeSTAR GXL DNA polymerase and $20 \mathrm{ng}$ of genomic DNA. The primer sequences were as follows: TRETpromF2, 5'-GCC GGG CTC CCA GTG GAT TCG-3' and the reverse primer TRETpromR2, 5'-GGC TTC CCA CGT GCG CAG CAG-3'. The PCR was conducted on a C-1000 Thermal Cycler (Bio-Rad Laboratories $\mathrm{AB}$ ) with an initial denaturation at $94^{\circ} \mathrm{C}$ for $30 \mathrm{sec}$, followed by 35 cycles of $7 \mathrm{sec}$ at $98^{\circ} \mathrm{C}, 90 \mathrm{sec}$ at $68^{\circ} \mathrm{C}$, and a final extension for $5 \mathrm{~min}$ at $68^{\circ} \mathrm{C}$. A total of $4 \mu \mathrm{l} \mathrm{PCR}$ products were stained with GelRed Nucleic Acid Gel Stain (Biotium Inc., Hayward, CA, USA), electrophoresed through a $1.0 \%$ agarose gel (certified molecular biology agarose (Bio-Rad Laboratories AB) and images for analysis were captured using a camera. The remaining amplified products were purified using the NucleoSpin Gel and PCR Clean-up kit followed by direct Sanger sequencing as above.

Methylation analysis. Methylation analysis of the $O-6-$ methylguanine-DNA methyltransferase $(M G M T)$ promoter was performed using the primers and PCR conditions described by Esteller et al (11). Methylation analysis of the cellular retinol binding protein $1(C R B P 1)$ promoter was performed using the primers and PCR conditions described by Jerónimo et al (11).

Total RNA and cDNA synthesis. Total RNA was extracted using an miRNeasy mini kit and QIAcube (Qiagen AB, Sollentuna, Sweden) according to the manufacturer's recommendations. Human Universal Reference Total RNA was used as a control (Clontech Laboratories, Inc., Mountainview, CA, USA); it is a mixture of total RNAs from male and female adult human tissues selected to represent a broad range of expressed genes. Reverse transcription (RT) was conducted as follows: 400-500 ng total RNA was reverse transcribed in a $20 \mu \mathrm{l}$ reaction volume using iScript Advanced cDNA Synthesis Kit for RT-qPCR according to the manufacturer's instructions (Bio-Rad Laboratories AB). Next, the cDNA was diluted to the equivalent of $10 \mathrm{ng} / \mu \mathrm{l}$ of RNA and $2 \mu \mathrm{l}$ was used as a template in subsequent PCR assays.

High-mobility group AT-hook 2 (HGMA2) expression analysis. To assess $H M G A 2$ expression in the periosteal chondroma tissue samples by PCR, the $25 \mu \mathrm{l}$ PCR volumes contained 12.5 $\mu 1$ Premix Taq polymerase (Takara Bio Europe SAS) $2 \mu \mathrm{l}$ diluted cDNA and $0.4 \mu \mathrm{M}$ of each of the forward and reverse primers (custom-made; Invitrogen Life Technologies). The PCRs were conducted on a C-1000 Thermal Cycler. The PCR conditions were as follows: An initial denaturation at $94^{\circ} \mathrm{C}$ for $30 \mathrm{sec}$ followed by 35 cycles of $7 \mathrm{sec}$ at $98^{\circ} \mathrm{C}$, $120 \mathrm{sec}$ at $68^{\circ} \mathrm{C}$, and a final extension for $5 \mathrm{~min}$ at $68^{\circ} \mathrm{C}$. The primer sequences used for the amplification of transcripts of HMGA2 exons 1-3 were as follows: HMGA2-846F1, 5'-CCA CTT CAG CCC AGG GAC AAC CT-3' and HMGA2-1021R, 5'-CCT CTT GGC CGT TTT TCT CCA GTG-3'. The quality of the cDNA synthesis was assessed by amplification of a cDNA fragment of the S100A10 gene, which is expressed in chondrocytes (12). The following primer sequences were used: 
S100A10-555F, 5'-TTC ACA AAT TCG CTG GGG ATA AAG G-3' and S100A10-840R, 5'-GAT TCC TTA AGC GAC CCT TTG GGA C-3'. The PCR for the amplification of transcripts of $H M G A 2$ were repeated three times. A total of $3 \mu 1$ PCR products were stained with the GelRed Nucleic Acid Gel Stain, electrophoresed through a $1.0 \%$ agarose gel and images for analysis were captured using a camera.

qPCR was also conducted to determine the expression level of $H M G A 2$. The TaqMan Gene Expression Assays Hs00171569_m1 (which probes HMGA2 exons 1-2), Hs00971725_m1 (which probes HMGA2 exons 4-5) and the S100A10 gene (control) assay Hs00237010_ml (Applied Biosystems Life Technologies, Foster City, CA, USA) were used. Four replicates of each sample and endogenous control were used to ensure reliability. The $20 \mu \mathrm{l}$ PCR reaction volume contained 1X TaqMan Universal Master Mix II with uracil n-glycosylase, 1X TaqMan gene expression mix, and cDNA (equivalent to 10 ng RNA). The PCR was run on the CFX96 Touch Real-Time PCR Detection System. The thermal cycling included an initial step at $50^{\circ} \mathrm{C}$ for $2 \mathrm{~min}$, followed by $10 \mathrm{~min}$ at $95^{\circ} \mathrm{C}, 40$ cycles of $15 \mathrm{sec}$ at $95^{\circ} \mathrm{C}$ and $1 \mathrm{~min}$ at $60^{\circ} \mathrm{C}$. The Bio-Rad CFX Manager software, version 2.1, was used to analyze the data and to calculate the mean quantification cycle (mean Cq).

\section{Results}

Clonal chromosomal aberrations. The clinical, cytogenetic and molecular analysis data are presented in Table I. The histologic examination of case 1 showing a nodular tumor of benign chondrocytes covered by periosteum is presented in Fig 1A. Clonal chromosomal aberrations were identified in two out of the four examined periosteal chondromas, while the other two tumors presented with a normal karyotype. Rearrangements of chromosome 12 q13-15 were observed in the two tumors with chromosome abnormalities (Table I). Case 3 has a simple balanced translocation between chromosomes 12 and $13, \mathrm{t}(12 ; 13)(\mathrm{q} 13 ; \mathrm{p} 11)$, as the sole aberration. The tumor in case 4 had two cytogenetically unrelated clones. The first clone displayed trisomy of chromosome 8 , while the second clone had numerical and structural aberrations, including a deletion in the long arm of chromosome 12, $\operatorname{del}(12)(\mathrm{q} 15 \mathrm{q} 23)$ (Table I, Fig. 1B).

Molecular genetic analyses. All four periosteal chondromas had the R132 mutation in $I D H 1$, whereas none had mutations in codon 172 of $I D H 2$ (Table I, Fig. 1C). Two tumors carried an IDH1R132C (CGT>TGT) mutation while the other two carried an IDH1R132L (CGT>CTT) mutation. None of the periosteal chondromas had methylated MGMT or CRBPI promoters or mutations at positions $-\mathrm{C} 228 \mathrm{~T}$ and $-\mathrm{C} 250 \mathrm{~T}$ in the promoter region of TERT (Table I).

The total RNA was extracted from the three tumors (cases 2-4) and a 310-bp S100A10 cDNA fragment was amplified in all of them, indicating that the synthesized cDNA was of good quality (Fig. 1D). RT-PCR with the primer set HMGA2-846F1/HMGA2-1021R did not amplify any cDNA fragments from the periosteal chondromas, whereas an amplified HMGA2 cDNA fragment was observed in the positive control (Fig. 1D). This result indicated that the HMGA2 
A

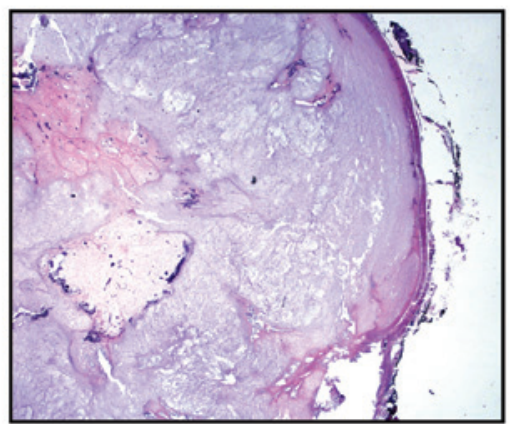

C

IDH1R132C

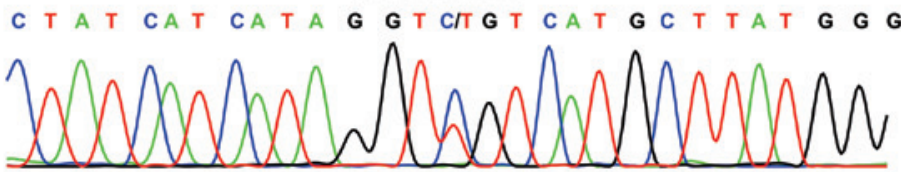

IDH1R132L

C T A T CA T CA T A G G T C T/GT CA T G C T T A T G G G G

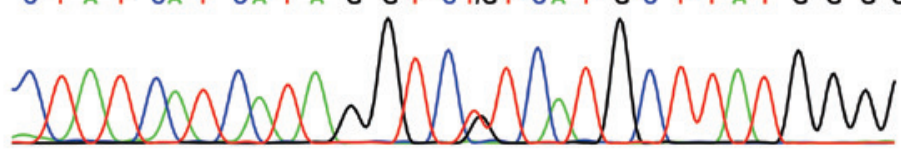

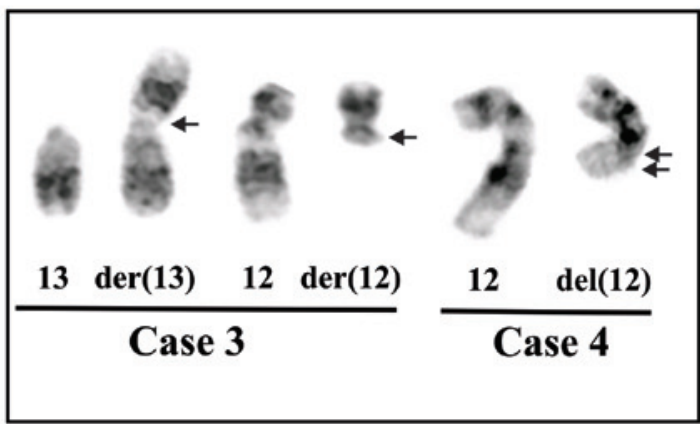

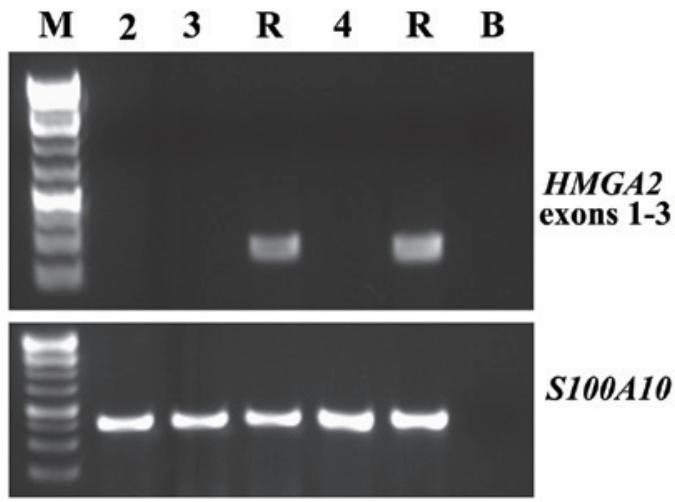

Figure 1. Histology, cytogenetics, HMGA2 expression, and IDH1 mutations in periosteal chondromas. (A) Histologic examination of case 1 exhibiting a nodular tumor of benign chondrocytes covered by periosteum. (B) Partial karyotypes presenting chromosome aberrations der(13)t(12;13)(q13;p11) and der(12) $\mathrm{t}(12 ; 13)(\mathrm{q} 13 ; \mathrm{p} 11)$ in case 3 , and $\operatorname{del}(12)(\mathrm{q} 15 \mathrm{q} 23)$ in case 4 , with the corresponding normal chromosome homologs; breakpoint positions are indicated by arrows. (C) Partial sequence chromatogram displaying the CGT>TGT (IDH1R132C; cases 1 and 2) and CGT>CTT in (IDH1R132L; cases 3 and 4) IDH1. (D) Polymerase chain reaction results demonstrating the amplification of HMGA2 exons 1-3 and S100A10. M, GeneRuler 1 Kb Plus DNA ladder (Thermo Fisher Scientific, Waltham, MA, USA); R, cDNA synthesized from Human Universal Reference Total RNA; B, no RNA in cDNA synthesis. HMGA2, high-mobility group AT-hook 2; IDH, isocitrate dehydrogenase.

transcript was not expressed in the three examined periosteal chondromas. Similar results were also obtained with qPCR: For the control Human Universal Reference Total RNA, the mean Cq was 25, 30 and 31 for S100A10, HMGA2 exons 1-2, and $H M G A 2$ exons 4-5, respectively (data not shown). The mean $\mathrm{Cq}$ for $S 100 A 10$ was 28,34 and 24 for cases 2, 3 and 4 , respectively, indicating that the gene expression assay was successful. However, there were no mean $\mathrm{Cq}$ values for HMGA2 exons 1-2/HMGA2 exons 4-5 since the TaqMan gene expression assays did not amplify any product (Table I). These results further indicate that $H G M A 2$ was not expressed in the periosteal chondromas examined in the present study.

\section{Discussion}

To the best of our knowledge, prior to the present study, karyotypic information on periosteal chondromas was restricted to seven cases and no consistent abnormality was recognized (4). Changes observed in periosteal chondromas in the previous study included loss of chromosome 6 and rearrangements of $2 q 37$, 4q21-25, 11q13-15 and 12q13 (4). The present study demonstrated that the $\mathrm{q}$ arm of chromosome 12 was involved in two out of the two periosteal chondromas with an informative karyotype.
Taken together, the data from the present study and those previously reported (3) demonstrate that rearrangements of 12q13-15 may be recurrent in this type of tumor. The involvement of the chromosome bands 12q13-15 in periosteal chondromas may not be random, particularly as they are frequently aberrant in benign connective tumors, such as lipoma and leiomyoma (14). In addition, 12-q rearrangements that result in the transcriptional activation of the HMGA2 gene, were reported in mesenchymal chondromas by Dahlén et al (15). They also demonstrated that $H M G A 2$ was expressed in four of six soft tissue chondromas, of which three tumors possessed a truncated (exons 1-3) transcript and one possessed the full-length (exons 1-5) transcript of $H M G A 2$. In addition, Dahlén et al (15) observed that HMGA2 was expressed in two skeletal chondromas: One of the tumors, which possessed a pericentric inversion of chromosome 12, expressed a truncated transcript of $H M G A 2$; whereas the other case, which had no visible involvement of $12 q$ by cytogenetic analysis, expressed the full-length $H M G A 2$ transcript (15). The description of these two tumors was inconclusive as to whether they were enchondromas or periosteal chondromas.

In the present study, neither conventional RT-PCR nor qPCR demonstrated expression of HMGA2 in the examined periosteal chondromas. 
The mutation analysis of $I D H I$ and $I D H 2$ revealed that the tumors carried heterozygous IDHI mutations at R132, which are in accordance with previous observations that a majority of periosteal chondromas carry heterozygous mutations at R132 of IDH1 $(5,6)$. From the results of the present study it can be hypothesized that the rearrangements and expression of $H M G A 2$ are mutually exclusive with $I D H 1$ and $I D H 2$ mutations in periosteal chondromas.

The R132L mutation in IDHI that was observed in two of the periosteal chondromas in the current study has also been identified previously by Amary et al (6), who observed this mutation in central low-grade cartilaginous tumors (1/23), chondrosarcomas GII and GIII (3/23) and dedifferentiated chondrosarcomas (2/13). Of the eight periosteal chondromas previously analyzed for the presence of mutations in $I D H 1$, mutations were observed in six: Four possessed IDH1R132C (CGT>TGT) and two possessed R132S (CGT>AGT) $(4,5)$. Viewing the present and previously published data in concert $(5,6)$, mutations in codon 12 of $I D H I$ have been present in the majority $(83 \%, 10 / 12)$ of examined periosteal chondromas. Of those mutations in the $I D H 1, \mathrm{R} 132 \mathrm{C}$ comprises 60\% (6/10), whereas R132L and R132S are observed in $20 \%$ ( 2 cases each). However, R132H, the most common mutation in gliomas, has not been reported thus far in periosteal chondromas. The exact role that $I D H I$ mutations serve in neoplasia is not fully understood, however it has been demonstrated that presence of a heterozygous R132H mutation induces genome-wide alterations in DNA methylation, leading to hypermethylation and hypomethylation $(16,17) . I D H 1$ mutations are associated with $M G M T$ and $C R B P 1$ promoter methylation in certain brain tumors (18-21). However, this association was not observed in the periosteal chondroma tissue specimens in the present study, since none of them possessed methylated MGMT or CRBP1 promoters, as determined using the MSP methodology $(11,12)$.

Mutations at positions $-\mathrm{C} 228 \mathrm{~T}$ and $-\mathrm{C} 250 \mathrm{~T}$ in the promoter region of TERT, which correspond to 124 and $146 \mathrm{bp}$ upstream of the TERT ATG start site, have been reported in gliomas and other tumors (10) but have yet to be studied in periosteal chondromas. TERT promoter mutations have been demonstrated to be inversely associated with $I D H 1 / I D H 2$ mutations in tumors of the nervous system $(22,23)$. In the present study, none of the four periosteal chondromas had $-\mathrm{C} 228 \mathrm{~T}$ or $-\mathrm{C} 250 \mathrm{~T}$ mutations. In conclusion, the present study revealed that the involvement of $12 \mathrm{q} 13-15$ in structural chromosomal aberrations is a relatively recurrent and common event in periosteal chondromas, that HMGA2 is not frequently expressed, that the majority of periosteal chondromas carry heterozygous IDH1R132 mutation, that MGMT and $C R B P 1$ promoters are not methylated, and that neither $-\mathrm{C} 228 \mathrm{~T}$ nor $-\mathrm{C} 250 \mathrm{~T}$ is present in the promoter region of TERT. Rearrangements of $H M G A 2$ resulting in fusion genes and expression of $H M G A 2$-fusion transcripts appear to be mutually exclusive with $I D H I$ and 2 mutations.

\section{Acknowledgements}

The authors thank Ms. Hege Brandt Gehrken for technical help. The present study was supported by grants from the Norwegian Cancer Society and the Norwegian Radium Hospital Foundation.

\section{References}

1. Lichtenstein L and Hall JE: Periosteal chondroma; a distinctive benign cartilage tumor. J Bone Joint Surg Am 34: 691-697, 1952.

2. Buddingh EP, Naumann S, Nelson M, Neffa JR, Birch N and Bridge JA: Cytogenetic findings in benign cartilaginous neoplasms. Cancer Genet Cytogenet 141: 164-168, 2003.

3. Mandahl N, Willén H, Rydholm A, Heim S and Mitelman F: Rearrangement of band q13 on both chromosomes 12 in a periosteal chondroma. Genes Chromosomes Cancer 6: 121-123, 1993.

4. Sakai Junior N, Abe KT, Formigli LM, et al: Cytogenetic findings in 14 benign cartilaginous neoplasms. Cancer Genet 204: 180-186, 2011.

5. Damato S, Alorjani M, Bonar F, et al: IDHI mutations are not found in cartilaginous tumours other than central and periosteal chondrosarcomas and enchondromas. Histopathology 60: 363-365, 2012.

6. Amary MF, Bacsi K, Maggiani F, et al: IDHland IDH2mutations are frequent events in central chondrosarcoma and central and periosteal chondromas but not in other mesenchymal tumours. J Pathol 224: 334-343, 2011

7. Mandahl N: Methods in solid tumour cytogenetics. In: Human cytogenetics: malignancy and acquired abnormalities. Rooney DE (ed). Oxford University Press, New York, NY, pp165-203, 2001.

8. Schaffer LG, Slovak ML and Campbell LJ (eds): ISCN 2009 an International System for Human Cytogenetic Nomenclature. Karger, Basel, 2009.

9. Patel KP, Barkoh BA, Chen Z, et al: Diagnostic testing for $I D H$ land $I D H 2$ variants in acute myeloid leukemia an algorithmic approach using high-resolution melting curve analysis. J Mol Diagn 13: 678-686, 2011.

10. Killela PJ, Reitman ZJ, Jiao Y, et al: TERT promoter mutations occur frequently in gliomas and a subset of tumors derived from cells with low rates of self-renewal. Proc Natl Acad Sci USA 110: 6021-6026, 2013

11. Esteller M, Hamilton SR, Burger PC, Baylin SB and Herman JG: Inactivation of the DNA repair gene O6-methylguanine-DNA methyltransferase by promoter hypermethylation is a common event in primary human neoplasia. Cancer Res 59: 793-797, 1999.

12. Jerónimo C, Henrique R, Oliveira J, et al: Aberrant cellular retinol binding protein $1(C R B P 1)$ gene expression and promoter methylation in prostate cancer. J Clin Pathol 57: 872-876, 2004.

13. Song C, Zhou X, Dong Q, et al: Regulation of inflammatory response in human chondrocytes by lentiviral mediated RNA interference against S100A10. Inflamm Res 61: 1219-1227, 2012.

14. Heim S and Mitelman F: Cancer Cytogenetics: Chromosomal and Molecular Genetic Abberations of Tumor Cells. 3rd edition. Wiley-Blackwell, Oxford, UK, 2009.

15. Dahlén A, Mertens F, Rydholm A, et al: Fusion, disruption, and expression of $H M G A 2$ in bone and soft tissue chondromas. Mod Pathol 16: 1132-1140, 2003.

16. Duncan CG, Barwick BG, Jin G, et al: A heterozygous IDH1R132H/WT mutation induces genome-wide alterations in DNA methylation. Genome Res 22: 2339-2355, 2012.

17. Turcan S, Rohle D, Goenka A, et al: IDHImutation is sufficient to establish the glioma hypermethylator phenotype. Nature 483 : 479-483, 2012.

18. Chou AP, Chowdhury R, Li S, et al: Identification of retinol binding protein 1 promoter hypermethylation in isocitrate dehydrogenase 1 and 2 mutant gliomas. J Natl Cancer Inst 104: 1458-1469, 2012

19. Leu S, von Felten S, Frank S, et al: IDH/MGMT-driven molecular classification of low-grade glioma is a strong predictor for long-term survival. Neuro Oncol 15: 469-479, 2013.

20. Mellai M, Monzeglio O, Piazzi A, et al: $M G M T$ promoter hypermethylation and its associations with genetic alterations in a series of 350 brain tumors. J Neurooncol 107: 617-631, 2012.

21. Noushmehr H, Weisenberger DJ, Diefes K, et al; Cancer Genome Atlas Research Network: Identification of a $\mathrm{CpG}$ island methylator phenotype that defines a distinct subgroup of glioma. Cancer Cell 17: 510-522, 2010.

22. Koelsche C, Sahm F, Capper D, et al: Distribution of TERT promoter mutations in pediatric and adult tumors of the nervous system. Acta Neuropathol 126: 907-915, 2013.

23. Nonoguchi N, Ohta T, Oh JE, Kim YH, Kleihues P and Ohgaki H: TERT promoter mutations in primary and secondary glioblastomas. Acta Neuropathol 126: 931-937, 2013. 